\title{
latrogenic injury of an aberrant right posterior sectoral bile duct
}

\section{John Cantrell, MB BCh, FCRad (Diag) SA}

Donald Gordon Medical Centre, University of the Witwatersrand, Johannesburg

Corresponding author: JCantrell (cantrell_j@hotmail.com)

\section{Case history}

A 34-year-old woman presented with a history of a previous laparoscopic cholecystectomy, followed within a few days by a formal laparotomy for a suspected bile duct injury. Approximately one week after the laparotomy, she developed a sinus on the anterior abdominal wall that was draining bile. She was then referred to our institution for further management. The earlier surgery was done at another hospital, and these details were not clear.

A CT scan, including a CT sinogram, was performed. The sinogram was done by inserting a catheter into the sinus and running in diluted contrast under gravity. CT images showed the sinus tract communicating with a collection in the gallbladder fossa, as well as contrast opacification of the segment 6 and 7 bile ducts. (Figs 1 and 2).

A week later, an endoscopic retrograde cholangiopancreatography (ERCP) examination was performed. This showed no filling of the right posterior sectoral ducts but normal opacification of the other ducts (Figs $3 \mathrm{a}$ and $\mathrm{b}$ ). These findings led to the diagnosis of an aberrant right posterior sectoral bile duct that was not identified prior to surgery and that was damaged at the time of laparoscopic cholecystectomy. This duct now drained into the gallbladder fossa, causing the collection and draining sinus.

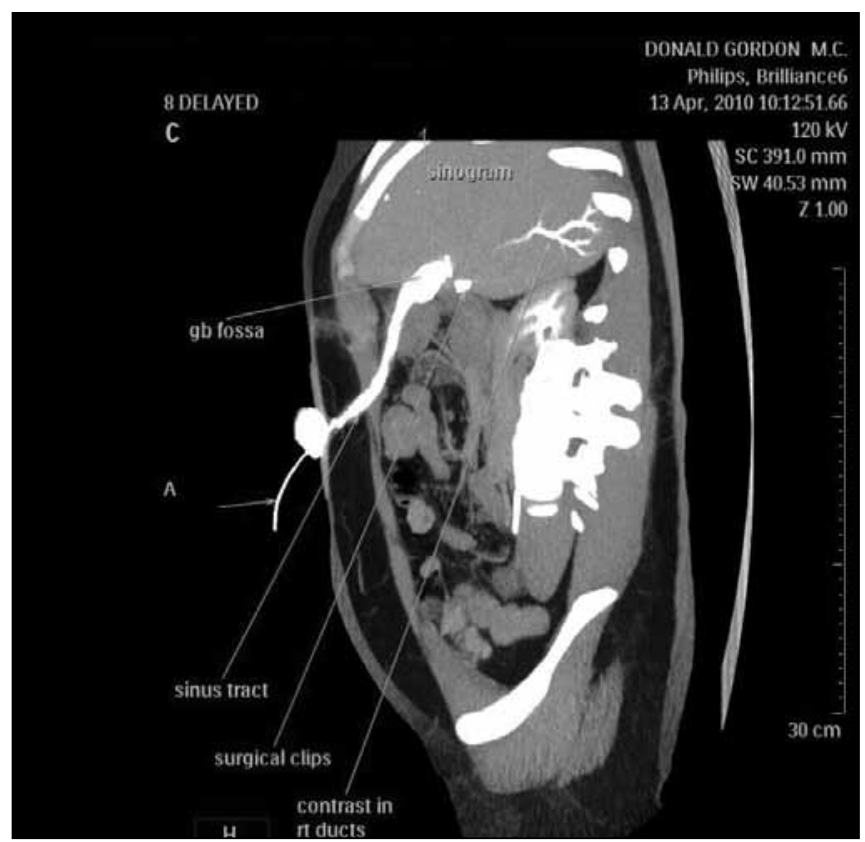

Fig. 1. Reconstructed $C T$ sinogram demonstrates contrast filling the sinus tract, gallbladder fossa and the right posterior sectoral ducts.

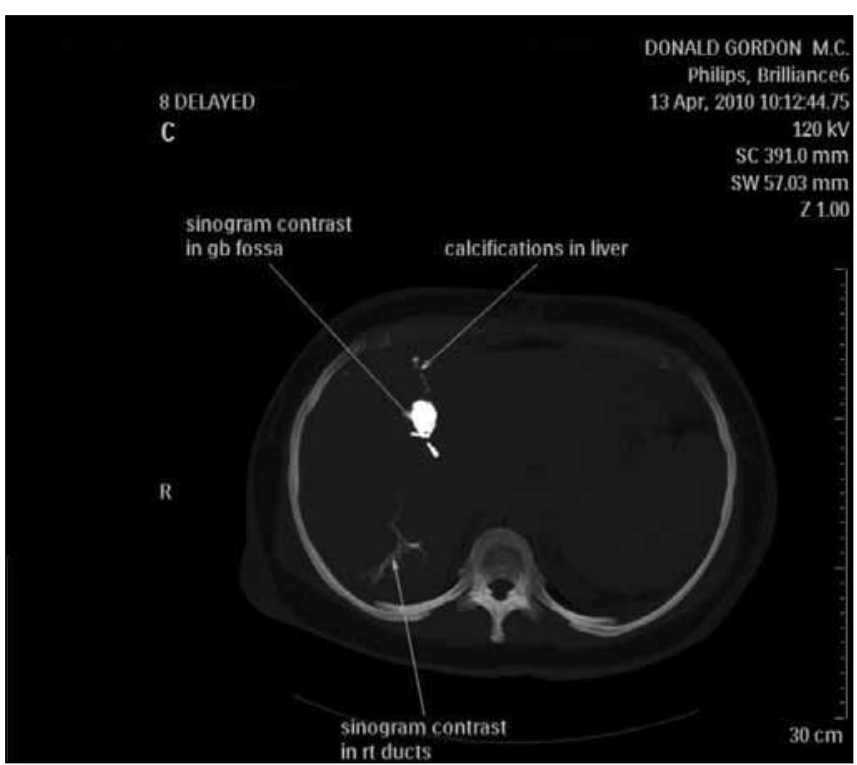

Fig. 2. Reconstructed CT sinogram with contrast in the gallbladder fossa and right posterior sectoral ducts (note surgical clips in the gallbladder fossa).

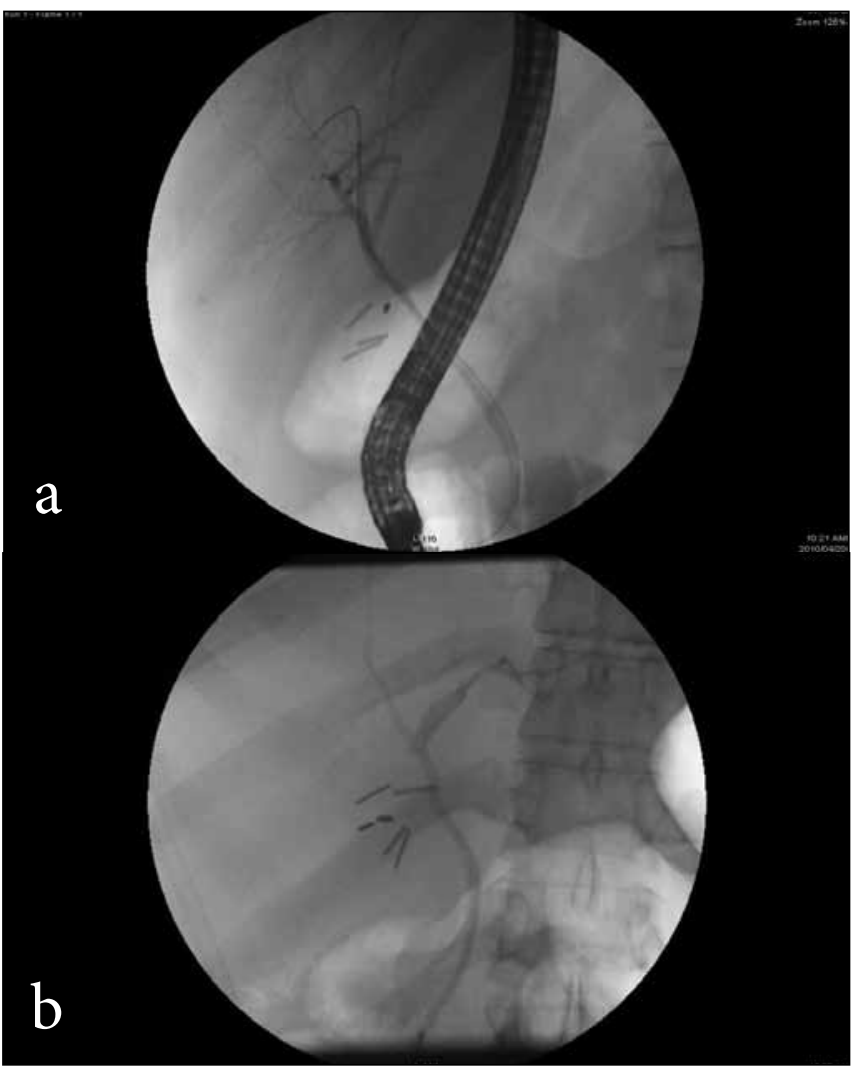

Figs $3 a, b$. ERCP shows normal left ducts and normal right anterior sectoral ducts, but non-filling of the right posterior sectoral ducts. 


\section{CASE REPORT}

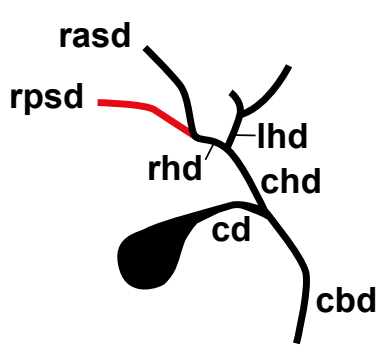

1.

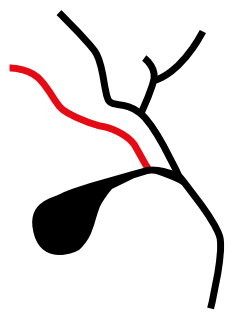

3.

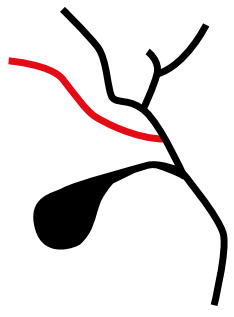

2.

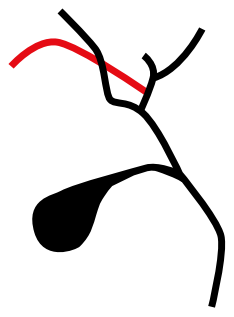

4.
Schematic diagrams demonstrating aberrant anatomy of the right posterior sectoral duct (RSPD) (shown in red).

1.) normal anatomy. 2.) insertion of the RPSD into the common hepatic duct. 3.) insertion of the RPSD into the cystic duct. 4.) insertion of the RPSD into the left hepatic duct.

cbd=common bile duct, cd=cystic duct, chd=common hepatic duct, rhd=right hepatic duct, Ind=left hepatic duct, rasd=right anterior sectoral duct, rpsd=right posterior sectoral duct.

Fig. 4. Anatomical variations of the right posterior sectoral bile duct.

\section{Discussion}

Pericholecystic biliary anomalies that are often overlooked at the time of laparoscopic cholecystectomy include both aberrant ducts and accessory ducts such as Luschka's duct anomaly (a meshwork of small ducts that extend from the right lobe of the liver in the gallbladder fossa to the right hepatic or common hepatic duct). ${ }^{1}$ The non-visualisation of the right posterior sectoral duct at ERCP supports the diagnosis of an aberrant duct as opposed to an accessory duct.

Bile duct injuries as a result of laparoscopic cholecystectomy are estimated to occur in $0.5 \%$ of cases and continue to exceed the rate at open cholecystectomy. Undiagnosed aberrant bile ducts are a common cause of bile duct injury. ${ }^{2}$

The incidence of aberrant biliary anatomy is approximately $13-25 \%$, with anomalies of the right ducts being the most frequent. ${ }^{2-4}$ Aberrant right sectoral ducts usually drain into the common hepatic or cystic duct (Fig. 4). Between 4.8 and $8.4 \%$ of the population have an aberrant right posterior sectoral duct draining segments 6 and $7 .^{2}$ Injuries to these ducts may be symptomatic as in our case, but are often asymptomatic, with atrophy of the affected segments over time.

1. Hirao K, Miyazaki A, Fujimoto T, Isomoto I, Hayashi K. Evaluation of aberrant bile ducts before laparoscopic cholecystectomy. AJR 2000; 175:713-720.

2. Babel N, Sakpal SV, Paragi P, Wellen J, Feldman S, Chamberlain RS. Iatrogenic bile duct injury associated with anomalies of the right hepatic sectoral ducts: a misunderstood and underappreciated problem. HPB Surg 2009; 2009: 153269.

3. Dahnert W. Radiology Review Manual. 6th ed. Philadelphia: Lippincott Williams and Wilkins, 2007: 687.

4. La Berge JM, Venbrux AC. Biliary Interventions. 1st ed. Fairfax, VA, USA: Society of Cardiovascular and Interventional Radiology, 1995: 42-43. 